\title{
RIEMANN BOUNDARY VALUE PROBLEM \\ FOR HYPERANALYTIC FUNCTIONS
}

\author{
RICARDO ABREU BLAYA, JUAN BORY REYES, AND DIXAN PEÑA PEÑA
}

Received 23 February 2005

We deal with Riemann boundary value problem for hyperanalytic functions. Furthermore, necessary and sufficient conditions for solvability of the problem are derived. At the end the explicit form of general solution for singular integral equations with a hypercomplex Cauchy kernel in the Douglis sense is established.

\section{Introduction}

The theory of Riemann boundary value problem for analytic functions of one complex variable and singular integral equations that are equivalent to it has been extensively studied in the literature. For classical books on this topic see $[7,12,13]$ and for an actual overview of them the reader is directed to the monograph by Estrada and Kanwal [6], and the references therein.

In the more recent times several generalizations and extensions of the theory are treated and have led to numerous important results not only for nonsmoothly bounded domain, which differs with the former, but for general assumptions on the data of the problem, such as generalized Hölder coefficients or special subspaces of this space and the desired boundary behavior condition for the solution. During the last decades, the Riemann boundary value problem was studied for generalized analytic functions, as well as for many other linear and nonlinear elliptic systems in the plane $[1,2,8,15,16,17]$.

Let $\gamma$ be a rectifiable positively oriented Jordan closed curve with diameter $d$ which is the boundary of a bounded simply connected domain $\Omega_{+}$in the complex plane $\mathbb{C}$ and let $\Omega_{-}:=\mathbb{C} \backslash\left(\Omega_{+} \cup \gamma\right)$.

In the Douglis commuting function algebra sense, a continuously differentiable null solution to the Douglis differential operator provides us with the class of hyperanalytic functions. Let $\mathscr{A}\left(\Omega_{ \pm}\right)$be the spaces of all continuous functions on $\overline{\Omega_{ \pm}}:=\Omega_{ \pm} \cup \gamma$ and hyperanalytic in $\Omega_{ \pm}$.

The classical Riemann boundary value problem for analytic functions consists in finding a function $\Phi(z)$ analytic in $\mathbb{C} \backslash \gamma$, such that $\Phi$ has a finite order at infinity, and satisfies a prescribed jump condition across the curve $\gamma$. The basic boundary condition takes 
2822 Riemann boundary value problem for hyperanalytic functions

the form

$$
\Phi^{+}(t)-G(t) \Phi^{-}(t)=g(t), \quad t \in \gamma
$$

where $G, g$ are given continuous functions on $\gamma$, and $\Phi^{+}(t)$ and $\Phi^{-}(t)$ represent the limit values, in a suitable sense, of the desired function $\Phi$ at a point $t$ as this point is approached from $\Omega_{+}$and from $\Omega_{-}$, respectively.

The Riemann boundary value problem for analytic functions as well as for hyperanalytic functions in the case where the unknown functions are continuous up to the boundary or their continuity is violated only at a finite number of points are well studied and described in the fundamental monographs $[7,8,12,13]$. The Riemann problems mentioned above are usually called a continuous or piecewise continuous boundary value problem, respectively.

The present paper is concerned with hyperanalytic Riemann boundary value problem (where instead of analyticity one requires the hyperanalyticity of $\Phi$ ) in the continuous case (the solutions including their boundary values on $\gamma$ are continuous). The purpose of the paper is to present an explicit form of the general solution of the problem.

The motivation comes on the one hand from the study of the hyperanalytic Riemann boundary value problem with continuous coefficients [10] and on the other from the necessary and sufficient solvability condition, which will be imposed on the layer function of the Cauchy type integral so that this integral provides the solution of the basic jump problem.

The main result is moreover applied to describe the general solution of a singular integral equation with a hypercomplex Cauchy kernel.

\section{Preliminaries}

For the sake of completeness we recall some basic notions and results in Douglis analysis, that is, a Douglis-algebra-valued function theory which is a generalization of classical complex analysis in the plane. For more details concerning this function theory and its application, we refer the reader to $[1,8,16,17]$.

Let $\mathbb{D}$ be the Douglis algebra generated by the elements $i$ and $e$. The multiplication in $\mathbb{D}$ is governed by the rules

$$
i^{2}=-1, \quad i e=e i, \quad e^{r}=0, \quad e^{0}=1,
$$

where $r$ is a positive integer.

An arbitrary element $a \in \mathbb{D}$ may be written as a hypercomplex number of the form

$$
a=\sum_{k=0}^{r-1} a_{k} e^{k}
$$

where each $a_{k}$ is a complex number, $a_{0}$ is called the complex part of $a$, and $A:=\sum_{k=1}^{r-1} a_{k} e^{k}$ is the nilpotent part. 
The conjugation $a \rightarrow \bar{a}$ in $\mathbb{D}$ is defined as

$$
\bar{a}:=\sum_{k=0}^{r-1} \bar{a}_{k} e^{k} .
$$

In $\mathbb{D}$ the algebraic norm of $a$ is defined by

$$
|a|:=\sum_{k=0}^{r-1}\left|a_{k}\right| .
$$

It is easily seen that

$$
|a b| \leq|a||b|, \quad|a+b| \leq|a|+|b|,
$$

for any hypercomplex numbers $a$ and $b$. The multiplicative inverse $a^{-1}$ of $a$ with complex part $a_{0} \neq 0$ is given by

$$
a^{-1} \text { or } \frac{1}{a}=\frac{1}{a_{0}} \sum_{k=0}^{r-1}(-1)^{k}\left(\frac{A}{a_{0}}\right)^{k} \text {. }
$$

Conversely, if $a_{0}=0$, then $a$ does not have a multiplicative inverse and $a$ is called nilpotent.

In what follows, we will consider functions $\mathbb{D}$-valued, which are defined in some subset $\Omega \subset \mathbb{C}$.

We say that $f=\sum_{k=0}^{r-1} f_{k} e^{k}$, where $f_{k}$ are complex-valued functions, belongs to some classical class of functions on $\Omega$ if each of its components $f_{k}$ belongs to that class.

The Douglis operator $\partial_{\bar{z}}^{q}$ is given by

$$
\partial_{\bar{z}}^{q}:=\partial_{\bar{z}}+q(z) \partial_{z}, \quad z=x+i y
$$

here $q(z)$ is a known nilpotent hypercomplex function and

$$
\partial_{\bar{z}}:=\frac{1}{2}\left(\partial_{x}+i \partial_{y}\right), \quad \partial_{z}:=\frac{1}{2}\left(\partial_{x}-i \partial_{y}\right)
$$

Definition 2.1. A continuously differentiable hypercomplex function $f$ is hyperanalytic in $\Omega$ if $\partial_{\bar{z}}^{q} f=0$ in $\Omega$.

The basic example of a hyperanalytic function is the generating solution of the Douglis operator given by

$$
W(z)=z+\sum_{k=1}^{r-1} W_{k}(z) e^{k},
$$

namely, $\partial_{\bar{z}}^{q} W(z)=0$ and its nilpotent part $\sum_{k=1}^{r-1} W_{k} e^{k}$ possess bounded and continuous derivatives up to order two in $\mathbb{C}$. 


\section{Riemann boundary value problem for hyperanalytic functions}

Since in the paper we have employed the letter $t$ to denote a generic point in the curve $\gamma$, we have decided to denote the generating solution of the Douglis operator with $W(z)$ instead of the standard notation $t(z)$.

The hypercomplex Cauchy kernel, that is, the fundamental solution of the Douglis operator, is given by

$$
2 e_{z}(\zeta):=\frac{1}{\pi} \frac{\partial_{\zeta} W(\zeta)}{W(\zeta)-W(z)}, \quad \zeta \neq z
$$

For $f, g \in \mathscr{C}^{1}\left(\Omega_{+}\right) \cap \mathscr{C}\left(\overline{\Omega_{+}}\right)$Green's identity can be formulated within the framework of hypercomplex function theory in the following way:

$$
\int_{\gamma} \partial_{\zeta} W(\zeta) f(\zeta) n_{q}(\zeta) g(\zeta) d s=2 \int_{\Omega_{+}} \partial_{\zeta} W(\zeta)\left(f \partial_{\bar{\zeta}}^{q} g+g \partial_{\bar{\zeta}}^{q} f\right) d \Omega_{+}
$$

where $n_{q}(\zeta):=n(\zeta)+\bar{n}(\zeta) q(\zeta), n(\zeta)$ denotes the exterior unit normal vector to $\gamma$ at the point $\zeta$, and $d s$ is an arc length differential.

Green's identity leads to the Cauchy-Pompeiu integral representation formula for smooth functions

$$
f(z)=\int_{\gamma} e_{z}(\zeta) n_{q}(\zeta) f(\zeta) d s-2 \int_{\Omega_{+}} e_{z}(\zeta) \partial_{\zeta}^{q} f(\zeta) d \Omega_{+}, \quad z \in \Omega_{+},
$$

while for hyperanalytic functions coincides with Cauchy's formula.

$$
f(z)=\int_{\gamma} e_{z}(\zeta) n_{q}(\zeta) f(\zeta) d s, \quad z \in \Omega_{+}
$$

Definition 2.2. Suppose $F$ is a hyperanalytic function outside of an open ball $B_{R}$ with radius $R>0$ and center at the origin and let $\gamma_{0}$ be any rectifiable positively oriented Jordan closed curve such that $\gamma_{0}$ lies in $\mathbb{C} \backslash \overline{B_{R}}$ and surrounds $\overline{B_{R}}$. The hypercomplex number

$$
\operatorname{Res}_{\zeta=\infty}[F(\zeta)]:=-\frac{1}{2 \pi} \int_{\gamma_{0}} W_{\zeta}(\zeta) n_{q}(\zeta) F(\zeta) d s
$$

is called the residue of $F$ at infinity.

Note that, according to (2.11), the integral in the right-hand side does not depend on the choice of the curve $\gamma_{0}$.

Theorem 2.3. Let $F \in \mathscr{A}\left(\Omega_{-}\right)$, then

$$
\int_{\gamma} e_{z}(\zeta) n_{q}(\zeta) F(\zeta) d s= \begin{cases}-\operatorname{Res}_{\zeta=\infty}\left[\frac{F(\zeta)}{W(\zeta)-W(z)}\right], & z \in \Omega_{+} \\ -F(z)-\operatorname{Res}_{\zeta=\infty}\left[\frac{F(\zeta)}{W(\zeta)-W(z)}\right], & z \in \Omega_{-}\end{cases}
$$


Proof. Suppose $z \in \Omega_{-}$and let $R>0$ such that $\Omega_{+} \subset B_{R}$ and $z \in B_{R}$. From Cauchy's formula we get

$$
\begin{aligned}
\int_{\gamma} e_{z}(\zeta) n_{q}(\zeta) F(\zeta) d s & =-F(z)+\int_{\partial B_{R}} e_{z}(\zeta) n_{q}(\zeta) F(\zeta) d s \\
& =-F(z)-\operatorname{Res}_{\zeta=\infty}\left[\frac{F(\zeta)}{W(\zeta)-W(z)}\right]
\end{aligned}
$$

The case $z \in \Omega_{+}$is similar.

Notation $c$ will be used for constants which may vary from one occurrence to the next; in general these constants only depend on $q$.

Lemma 2.4. Let $\gamma_{\epsilon}(t):=\{\zeta \in \gamma:|\zeta-t| \leq \epsilon\}$, for $t \in \gamma$ and let $\epsilon \in(0, d]$.

(i) If $F \in \mathscr{A}\left(\Omega_{+}\right)$, then

$$
\left|\int_{\gamma \backslash \gamma_{\epsilon}(t)} e_{t}(\zeta) n_{q}(\zeta)(F(\zeta)-F(t)) d s\right| \leq c \max _{z \in \Omega_{+},|z-t|=\epsilon}|F(z)-F(t)| .
$$

(ii) If $F \in \mathscr{A}\left(\Omega_{-}\right)$, then

$$
\begin{aligned}
& \left|\int_{\gamma \backslash \gamma_{\epsilon}(t)} e_{t}(\zeta) n_{q}(\zeta)(F(\zeta)-F(t)) d s+F(t)+\operatorname{Res} \zeta=\infty\left[\frac{F(\zeta)}{W(\zeta)-W(t)}\right]\right| \\
& \quad \leq c \max _{z \in \Omega_{-},|z-t|=\epsilon}|F(z)-F(t)| .
\end{aligned}
$$

Proof. The first assertion was already proved in [10]. We consider the second one. Suppose $B_{\epsilon}(t)$ is the open ball with center $t$ and radius $\epsilon$, and let

$$
Q:=\Omega_{+} \cup B_{\epsilon}(t)
$$

Since $\gamma \backslash \gamma_{\epsilon}(t)=\partial Q \backslash\left(\partial Q \cap \partial B_{\epsilon}(t)\right)$, we have

$$
\begin{aligned}
\int_{\gamma \backslash \gamma_{\epsilon}(t)} & e_{t}(\zeta) n_{q}(\zeta)(F(\zeta)-F(t)) d s \\
= & \int_{\partial Q} e_{t}(\zeta) n_{q}(\zeta)(F(\zeta)-F(t)) d s-\int_{\partial Q \cap \partial B_{\epsilon}(t)} e_{t}(\zeta) n_{q}(\zeta)(F(\zeta)-F(t)) d s \\
= & \int_{\partial Q} e_{t}(\zeta) n_{q}(\zeta) F(\zeta) d s-F(t)-\int_{\partial Q \cap \partial B_{\epsilon}(t)} e_{t}(\zeta) n_{q}(\zeta)(F(\zeta)-F(t)) d s .
\end{aligned}
$$

In view of the previous theorem,

$$
\begin{aligned}
& \left|\int_{\gamma \backslash \gamma_{\epsilon}(t)} e_{t}(\zeta) n_{q}(\zeta)(F(\zeta)-F(t)) d s+F(t)+\operatorname{Res} \zeta=\infty\left[\frac{F(\zeta)}{W(\zeta)-W(t)}\right]\right| \\
& =\left|\int_{\partial Q \cap \partial B_{\epsilon}(t)} e_{t}(\zeta) n_{q}(\zeta)(F(\zeta)-F(t)) d s\right| \leq c \max _{z \in \overline{\Omega_{-}},|z-t|=\epsilon}|F(z)-F(t)| .
\end{aligned}
$$


2826 Riemann boundary value problem for hyperanalytic functions

For each $f \in \mathscr{C}(\gamma)$ the Cauchy-type integral is given by

$$
\left(\mathbf{C}_{\gamma} f\right)(z):=\int_{\gamma} e_{z}(\zeta) n_{q}(\zeta) f(\zeta) d s, \quad z \notin \gamma
$$

Clearly $\mathbf{C}_{\gamma} f$ is hyperanalytic in $\mathbb{C} \backslash \gamma$.

Thus the singular Cauchy integral operator (Hilbert transform) on $\gamma$ reads as

$$
\left(\mathbf{S}_{\gamma} f\right)(t):=2 \int_{\gamma} e_{t}(\zeta) n_{q}(\zeta)(f(\zeta)-f(t)) d s+f(t), \quad t \in \gamma
$$

where the integral which defines $\mathbf{S}_{\gamma} f$ has to be taken in the sense of Cauchy's principal value, and the function $f$ is such that the integrals

$$
\int_{\gamma_{\epsilon}(t)} e_{t}(\zeta) n_{q}(\zeta)(f(\zeta)-f(t)) d s
$$

converge uniformly to zero for $t \in \gamma$ as $\epsilon \rightarrow 0$.

The space of all continuous functions on $\gamma$ which satisfy the above condition will be denoted by $\mathscr{S}(\gamma)$. Taking into account Lemma 2.4 , we get $\mathscr{A}\left(\Omega_{ \pm}\right) \subset \mathscr{S}(\gamma)$.

From now on we always assume $\gamma$ to be a regular closed curve, that is, the quotient of the length of $\gamma$ inside any circle to the radius of the circle is less than some fixed constant.

In a previous paper [3] we already studied the problem of establishing necessary and sufficient condition for the Cauchy-type integral to be continuously extended onto $\gamma$. The following theorem was proved in [3].

Theorem 2.5. Let $f \in \mathscr{C}(\gamma)$, then $\mathbf{C}_{\gamma} f$ has continuous limit values on $\gamma$ if and only if $f \in \mathscr{S}(\gamma)$. Moreover,

$$
\lim _{\Omega_{ \pm} \ni z \rightarrow t}\left(\mathbf{C}_{\gamma} f\right)(z)=\frac{1}{2}\left(\left(\mathbf{S}_{\gamma} f\right)(t) \pm f(t)\right)
$$

Therefore, for any $f \in \mathscr{S}(\gamma)$ the functions

$$
\left(\mathbf{C}_{\gamma}^{ \pm} f\right)(z):= \begin{cases}\left(\mathbf{C}_{\gamma} f\right)(z), & z \in \Omega_{ \pm}, \\ \frac{1}{2}\left(\left(\mathbf{S}_{\gamma} f\right)(z) \pm f(z)\right), & z \in \gamma,\end{cases}
$$

are continuous on $\overline{\Omega_{ \pm}}$.

Let $\mathscr{S}^{ \pm}(\gamma)$ be the spaces of all continuous functions $f$ on $\gamma$ which have hyperanalytic extensions $f^{ \pm}$to $\Omega_{ \pm}$and $f^{-}(\infty)=0$.

By Theorem 2.5 we get the splitting of the space $\mathscr{S}(\gamma)$ as

$$
\mathscr{S}(\gamma)=\mathscr{S}^{+}(\gamma) \oplus \mathscr{S}^{-}(\gamma)
$$

and the corresponding splitting of the functions considered $f=f^{+}+f^{-}$, where $f^{ \pm} \in$ $\mathscr{S} \pm(\gamma)$. 
Now, for $f \in \mathscr{C}(\gamma)$ we consider the modulus of continuity for $f$ as

$$
\omega_{f}(\xi):=\sup _{\rho \geq \xi} \rho^{-1} \max _{t_{1}, t_{2} \in \gamma,\left|t_{1}-t_{2}\right| \leq \rho}\left|f\left(t_{1}\right)-f\left(t_{2}\right)\right|, \quad \xi>0
$$

Let

$$
I_{0}(\gamma):=\left\{f \in \mathscr{C}(\gamma): \int_{0}^{d} \frac{\omega_{f}(\xi)}{\xi} d \xi<+\infty\right\}
$$

For a majorant $\varphi$, that is, $\varphi$ is a positive real function on $(0, d]$ such that $\varphi(\xi)$ does not decrease, $\varphi(\xi) / \xi$ does not increase, and $\varphi(\xi) \rightarrow 0$ as $\xi \rightarrow 0$, we now introduce the generalized Hölder continuous functions space

$$
\mathscr{H}_{\varphi}(\gamma):=\left\{f \in \mathscr{C}(\gamma): \omega_{f}(\xi) \leq c \varphi(\xi), \xi \in(0, d]\right\}
$$

and its subspace

$$
\mathscr{L}_{\varphi}(\gamma):=\left\{f \in \mathscr{H}_{\varphi}(\gamma): \Theta_{f}(\xi) \leq c \varphi(\xi), \xi \in(0, d]\right\}
$$

where

$$
\Theta_{f}(\xi):=\xi \sup _{\rho \geq \xi} \rho^{-1} \sup _{\epsilon \in(0, \rho], t \in \gamma}\left|\int_{\gamma_{\epsilon}(t)} e_{t}(\zeta) n_{q}(\zeta)(f(\zeta)-f(t)) d s\right|
$$

One can define a norm in $\mathscr{L}_{\varphi}(\gamma)$ by

$$
\|f\|_{\mathscr{L}_{\varphi}}:=\max _{t \in \gamma}|f(t)|+\sup _{\xi \in(0, d]} \frac{\omega_{f}(\xi)}{\varphi(\xi)}+\sup _{\xi \in(0, d]} \frac{\Theta_{f}(\xi)}{\varphi(\xi)}
$$

In the sequel we will make use of the following two technical lemmas, from which other results will follow.

Lemma 2.6. Let $f \in \mathscr{S}(\gamma)$, then for $t \in \gamma$ and $\epsilon \in(0, d]$,

$$
\sup _{z \in \overline{\Omega_{ \pm}},|z-t|=\epsilon}\left|\left(\mathbf{C}_{\gamma}^{ \pm} f\right)(z)-\left(\mathbf{C}_{\gamma}^{ \pm} f\right)(t)\right| \leq c\left(\omega_{f}(\epsilon)+\Theta_{f}(\epsilon)+\epsilon \int_{\epsilon}^{d} \frac{\omega_{f}(\xi)}{\xi^{2}} d \xi\right)
$$


2828 Riemann boundary value problem for hyperanalytic functions

Proof. It is only necessary to distinguish the following two cases.

Case 1. $z \in \Omega_{ \pm}$. Let $t_{z} \in \gamma$ such that $\left|z-t_{z}\right|=\operatorname{dist}(z, \gamma)=: \nu$. Then for $|z-t|=\epsilon$,

$$
\begin{aligned}
& \left(\mathbf{C}_{\gamma} f\right)(z)-\left(\mathbf{C}_{\gamma}^{ \pm} f\right)(t) \\
& =\int_{\gamma_{\nu}\left(t_{z}\right)} e_{z}(\zeta) n_{q}(\zeta)\left(f(\zeta)-f\left(t_{z}\right)\right) d s \\
& \quad+\int_{\gamma \backslash \gamma_{\nu}\left(t_{z}\right)}\left(e_{z}(\zeta)-e_{t_{z}}(\zeta)\right) n_{q}(\zeta)\left(f(\zeta)-f\left(t_{z}\right)\right) d s \\
& \quad-\int_{\gamma_{\nu}\left(t_{z}\right)} e_{t_{z}}(\zeta) n_{q}(\zeta)\left(f(\zeta)-f\left(t_{z}\right)\right) d s+\frac{1}{2}\left(\left(\mathbf{S}_{\gamma} f\right)\left(t_{z}\right)-\left(\mathbf{S}_{\gamma} f\right)(t)\right) \\
& \quad \pm \frac{1}{2}\left(f\left(t_{z}\right)-f(t)\right)=: \sum_{k=1}^{5} J_{k} .
\end{aligned}
$$

Because $\left|z-t_{z}\right| \leq|\zeta-z|$ and $\left|\zeta-t_{z}\right| \leq|\zeta-z|+\left|z-t_{z}\right| \leq 2|\zeta-z|$,

$$
\begin{aligned}
\left|J_{1}\right|+\left|J_{2}\right| & \leq c\left(\int_{\gamma_{\nu}\left(t_{z}\right)} \frac{\omega_{f}\left(\left|\zeta-t_{z}\right|\right)}{|\zeta-z|} d s+\left|z-t_{z}\right| \int_{\gamma \backslash \gamma_{\nu}\left(t_{z}\right)} \frac{\omega_{f}\left(\left|\zeta-t_{z}\right|\right)}{|\zeta-z|\left|\zeta-t_{z}\right|} d s\right) \\
& \leq c\left(\omega_{f}(\nu)+\left|z-t_{z}\right| \int_{\gamma \backslash \gamma_{\nu}\left(t_{z}\right)} \frac{\omega_{f}\left(\left|\zeta-t_{z}\right|\right)}{\left|\zeta-t_{z}\right|^{2}} d s\right) \\
& \leq c\left(\omega_{f}(\nu)+\nu \int_{\nu}^{d} \frac{\omega_{f}(\xi)}{\xi^{2}} d \xi\right) .
\end{aligned}
$$

First, we remark that the second term in the last inequality is an almost-increasing function in $v$. There is no loss of generality in assuming that $\nu \leq \epsilon \leq d / 2$, then

$$
\begin{aligned}
& 3 \epsilon \int_{\epsilon}^{d} \frac{\omega_{f}(\xi)}{\xi^{2}} d \xi-\nu \int_{\nu}^{d} \frac{\omega_{f}(\xi)}{\xi^{2}} d \xi \\
& \quad=(\epsilon-\nu) \int_{\epsilon}^{d} \frac{\omega_{f}(\xi)}{\xi^{2}} d \xi+\left(2 \epsilon \int_{\epsilon}^{d} \frac{\omega_{f}(\xi)}{\xi^{2}} d \xi-\nu \int_{\nu}^{\epsilon} \frac{\omega_{f}(\xi)}{\xi^{2}} d \xi\right) .
\end{aligned}
$$

Further, it is clear that

$$
2 \epsilon \int_{\epsilon}^{d} \frac{\omega_{f}(\xi)}{\xi^{2}} d \xi \geq 2 \epsilon \omega_{f}(\epsilon) \int_{\epsilon}^{d} \frac{d \xi}{\xi^{2}}=2 \epsilon \omega_{f}(\epsilon) \frac{d-\epsilon}{\epsilon d}=2 \omega_{f}(\epsilon) \frac{d-\epsilon}{d} \geq \omega_{f}(\epsilon)
$$

On the other hand,

$$
\nu \int_{\nu}^{\epsilon} \frac{\omega_{f}(\xi)}{\xi^{2}} d \xi \leq \nu \omega_{f}(\epsilon) \int_{\nu}^{\epsilon} \frac{d \xi}{\xi^{2}}=v \omega_{f}(\epsilon) \frac{\epsilon-\nu}{\nu \epsilon}=\omega_{f}(\epsilon) \frac{\epsilon-\nu}{\epsilon} \leq \omega_{f}(\epsilon)
$$


and hence

$$
3 \epsilon \int_{\epsilon}^{d} \frac{\omega_{f}(\xi)}{\xi^{2}} d \xi-v \int_{\nu}^{d} \frac{\omega_{f}(\xi)}{\xi^{2}} d \xi \geq 0
$$

It then follows that

$$
\left|J_{1}\right|+\left|J_{2}\right| \leq c\left(\omega_{f}(\epsilon)+\epsilon \int_{\epsilon}^{d} \frac{\omega_{f}(\xi)}{\xi^{2}} d \xi\right)
$$

For $J_{3}$ we obtain

$$
\left|J_{3}\right| \leq \Theta_{f}(\nu) \leq \Theta_{f}(\epsilon)
$$

Since $\left|t_{z}-t\right| \leq\left|t_{z}-z\right|+|z-t| \leq 2|z-t|=2 \epsilon$, then

$$
\left|J_{4}\right| \leq \omega_{\mathbf{S}_{\gamma} f}(\epsilon), \quad\left|J_{5}\right| \leq \omega_{f}(\epsilon) .
$$

On the other hand, starting from the reasonings in [3, Theorem 2] and using the definition of $\Theta_{f}$, we get

$$
\left|J_{4}\right| \leq \omega_{\mathbf{s}_{\gamma} f}(\epsilon) \leq c\left(\omega_{f}(\epsilon)+\Theta_{f}(\epsilon)+\epsilon \int_{\epsilon}^{d} \frac{\omega_{f}(\xi)}{\xi^{2}} d \xi\right) .
$$

Therefore

$$
\left|\left(\mathbf{C}_{\gamma} f\right)(z)-\left(\mathbf{C}_{\gamma}^{ \pm} f\right)(t)\right| \leq c\left(\omega_{f}(\epsilon)+\Theta_{f}(\epsilon)+\epsilon \int_{\epsilon}^{d} \frac{\omega_{f}(\xi)}{\xi^{2}} d \xi\right)
$$

Case 2. $z \in \gamma$. If $|z-t|=\epsilon$, then

$$
\left(\mathbf{C}_{\gamma}^{ \pm} f\right)(z)-\left(\mathbf{C}_{\gamma}^{ \pm} f\right)(t)=\frac{1}{2}\left(\mathbf{S}_{\gamma} f(z)-\mathbf{S}_{\gamma} f(t)\right) \pm \frac{1}{2}(f(z)-f(t)) .
$$

For that

$$
\left|\left(\mathbf{C}_{\gamma}^{ \pm} f\right)(z)-\left(\mathbf{C}_{\gamma}^{ \pm} f\right)(t)\right| \leq \omega_{\mathbf{S}_{\gamma} f}(\epsilon)+\omega_{f}(\epsilon)
$$

hence

$$
\left|\left(\mathbf{C}_{\gamma}^{ \pm} f\right)(z)-\left(\mathbf{C}_{\gamma}^{ \pm} f\right)(t)\right| \leq c\left(\omega_{f}(\epsilon)+\Theta_{f}(\epsilon)+\epsilon \int_{\epsilon}^{d} \frac{\omega_{f}(\xi)}{\xi^{2}} d \xi\right)
$$

The statement of the lemma follows now from the above-considered cases. 
2830 Riemann boundary value problem for hyperanalytic functions

Lemma 2.7. Suppose $f \in \mathscr{S}(\gamma)$ and let $\epsilon \in(0, d]$. Then

$$
\Theta_{S_{\gamma} f}(\epsilon) \leq c\left(\omega_{f}(\epsilon)+\Theta_{f}(\epsilon)+\epsilon \int_{\epsilon}^{d} \frac{\omega_{f}(\xi)}{\xi^{2}} d \xi\right)
$$

Proof. For $t \in \gamma$ we have $\left(\mathbf{S}_{\gamma} f\right)(t)=2\left(\mathbf{C}_{\gamma}^{+} f\right)(t)-f(t)$. Therefore

$$
\begin{aligned}
& \left|\int_{\gamma_{\epsilon}(t)} e_{t}(\zeta) n_{q}(\zeta)\left(\left(\mathbf{S}_{\gamma} f\right)(\zeta)-\left(\mathbf{S}_{\gamma} f\right)(t)\right) d s\right| \\
& \quad \leq \Theta_{f}(\epsilon)+2\left|\int_{\gamma_{\epsilon}(t)} e_{t}(\zeta) n_{q}(\zeta)\left(\left(\mathbf{C}_{\gamma}^{+} f\right)(\zeta)-\left(\mathbf{C}_{\gamma}^{+} f\right)(t)\right) d s\right|
\end{aligned}
$$

As a consequence of Lemma 2.4 we have

$$
\int_{\gamma} e_{t}(\zeta) n_{q}(\zeta)\left(\left(\mathbf{C}_{\gamma}^{+} f\right)(\zeta)-\left(\mathbf{C}_{\gamma}^{+} f\right)(t)\right) d s=0
$$

for that

$$
\begin{aligned}
& \left|\int_{\gamma_{\epsilon}(t)} e_{t}(\zeta) n_{q}(\zeta)\left(\left(\mathbf{C}_{\gamma}^{+} f\right)(\zeta)-\left(\mathbf{C}_{\gamma}^{+} f\right)(t)\right) d s\right| \\
& =\left|\int_{\gamma \backslash \gamma_{\epsilon}(t)} e_{t}(\zeta) n_{q}(\zeta)\left(\left(\mathbf{C}_{\gamma}^{+} f\right)(\zeta)-\left(\mathbf{C}_{\gamma}^{+} f\right)(t)\right) d s\right|
\end{aligned}
$$

Thus, returning to the Lemma 2.4, we obtain

$$
\left|\int_{\gamma_{\epsilon}(t)} e_{t}(\zeta) n_{q}(\zeta)\left(\left(\mathbf{C}_{\gamma}^{+} f\right)(\zeta)-\left(\mathbf{C}_{\gamma}^{+} f\right)(t)\right) d s\right| \leq c \sup _{z \in \overline{\Omega_{+}},|z-t|=\epsilon}\left|\left(\mathbf{C}_{\gamma}^{+} f\right)(z)-\left(\mathbf{C}_{\gamma}^{+} f\right)(t)\right|
$$

and the following estimate follows in view of the result of Lemma 2.6:

$$
\left|\int_{\gamma_{\epsilon}(t)} e_{t}(\zeta) n_{q}(\zeta)\left(\left(\mathbf{C}_{\gamma}^{+} f\right)(\zeta)-\left(\mathbf{C}_{\gamma}^{+} f\right)(t)\right) d s\right| \leq c\left(\omega_{f}(\epsilon)+\Theta_{f}(\epsilon)+\epsilon \int_{\epsilon}^{d} \frac{\omega_{f}(\xi)}{\xi^{2}} d \xi\right)
$$

Finally

$$
\left|\int_{\gamma_{\epsilon}(t)} e_{t}(\zeta) n_{q}(\zeta)\left(\left(\mathbf{S}_{\gamma} f\right)(\zeta)-\left(\mathbf{S}_{\gamma} f\right)(t)\right) d s\right| \leq c\left(\omega_{f}(\epsilon)+\Theta_{f}(\epsilon)+\epsilon \int_{\epsilon}^{d} \frac{\omega_{f}(\xi)}{\xi^{2}} d \xi\right)
$$

This completes the proof. 
The following theorem gives sufficient condition for the singular Cauchy integral operator to be bounded on $\mathscr{L}_{\varphi}(\gamma)$.

Theorem 2.8. Suppose $\varphi$ is a majorant such that for $\epsilon \in(0, d]$,

$$
\epsilon \int_{\epsilon}^{d} \frac{\varphi(\xi)}{\xi^{2}} d \xi \leq c \varphi(\epsilon)
$$

Then the singular Cauchy integral operator $\mathbf{S}_{\gamma}$ is a bounded operator on $\mathscr{E}_{\varphi}(\gamma)$, that is,

$$
\left\|\mathbf{S}_{\gamma} f\right\|_{\mathscr{F}_{\varphi}} \leq c\|f\|_{\mathscr{L}_{\varphi}}
$$

for any $f \in \mathscr{E}_{\varphi}(\gamma)$.

Proof. Suppose $f \in \mathscr{L}_{\varphi}(\gamma)$, then for $\epsilon \in(0, d]$,

$$
\omega_{f}(\epsilon) \leq\|f\|_{\mathscr{L}_{\varphi}} \varphi(\epsilon), \quad \Theta_{f}(\epsilon) \leq\|f\|_{\mathscr{F}_{\varphi}} \varphi(\epsilon) ;
$$

hence

$$
\epsilon \int_{\epsilon}^{d} \frac{\omega_{f}(\xi)}{\xi^{2}} d \xi \leq \epsilon\|f\|_{\mathscr{L}_{\varphi}} \int_{\epsilon}^{d} \frac{\varphi(\xi)}{\xi^{2}} d \xi \leq c\|f\|_{\mathscr{L}_{\varphi}} \varphi(\epsilon)
$$

Combining the previous estimates with Lemma 2.7, we obtain

$$
\Theta_{\mathrm{S}_{\gamma} f}(\epsilon) \leq c\|f\|_{\mathscr{L}_{\varphi}} \varphi(\epsilon)
$$

and similarly

$$
\omega_{\mathbf{S}_{\gamma} f}(\epsilon) \leq c\|f\|_{\mathscr{L}_{\varphi}} \varphi(\epsilon)
$$

So we have at once $\mathbf{S}_{\gamma} f \in \mathscr{L}_{\varphi}(\gamma)$. Besides

$$
\left|\mathbf{S}_{\gamma} f(t)\right| \leq 2 \Theta_{f}(d)+\|f\|_{\mathscr{L}_{\varphi}} \leq c\|f\|_{\mathscr{L}_{\varphi}}, \quad t \in \gamma,
$$

thus

$$
\left\|\mathbf{S}_{\gamma} f\right\|_{\mathscr{F}_{\varphi}} \leq c\|f\|_{\mathscr{L}_{\varphi}}
$$

that is, $\mathbf{S}_{\gamma}$ is a bounded operator on $\mathscr{L}_{\varphi}(\gamma)$.

Notice that the above theorem is a generalization of an analogous result by Bustamante and González in [11] treated in the framework of complex analysis.

\section{Riemann boundary value problem}

For the Riemann boundary value problem for hyperanalytic functions, due to the technique of canonical factorization, the interested reader can find a good introduction in [8]. 


\section{Riemann boundary value problem for hyperanalytic functions}

At the same time early quite more-general results on complete solution of the Riemann problem for hypercomplex functions, in case where the integration curve is rectifiable and the coefficients are assumed to be in special subspace of continuous function space, have been obtained in $[10,18]$.

Systematically the study of the solvability of the Riemann boundary problem for analytic functions is technically involved with the Cauchy-type integral which, by taking boundary values, led to the Sokhotski-Plemelj formulas. In such way the singular Cauchy integral operator appearing in these formulas transforms the Riemann problem to a singular integral equation. A good presentation for excellent examples of such study which is close to the present paper could be $[4,5,14]$.

The main goal of this paper is to develop a theory of the well-posed continuous Riemann boundary value problem by assuming that the given continuous coefficients defined on $\gamma$ have to agree with the desired boundary behavior of the solutions, that is, the solutions including their boundary values on $\gamma$ are continuous functions too.

The results of boundedness of the singular Cauchy integral operator and on the continuous extension of the Cauchy-type integral, as established in the previous section, are now applied to obtain a necessary and sufficient condition for the solvability of the Riemann problem with coefficient $G$ in the case where $G$ admits a canonical factorization

$$
G=\frac{H^{+}}{H^{-}}
$$

where $H^{ \pm} \in \mathscr{A}\left(\Omega_{ \pm}\right)$and the complex parts $H_{0}^{ \pm}$never vanish on $\overline{\Omega_{ \pm}}$.

Take an arbitrary fixed point $z_{0}$ in $\Omega_{+}$. We restrict ourselves to the case of a hypercomplex function $G$ which has complex part $G_{0}$ and never vanishes on $\gamma$. From this assumption the integer

$$
\kappa:=\frac{1}{2 \pi}\left[\arg G_{0}(\zeta)\right]_{\gamma}
$$

has significant importance and is called the index of $G$ with respect to $\gamma$, also called index of the Riemann boundary value problem. Note that the index of the function $(W(\zeta)-$ $\left.W\left(z_{0}\right)\right)^{\kappa}$ with respect to $\gamma$ is $\kappa$, and hence the index of $\left(W(\zeta)-W\left(z_{0}\right)\right)^{-\kappa} G(\zeta)$ is zero.

We may verify directly that

$$
X(z):= \begin{cases}\exp \Gamma(z), & z \in \Omega_{+}, \\ \left(W(z)-W\left(z_{0}\right)\right)^{-\kappa} \exp \Gamma(z), & z \in \Omega_{-},\end{cases}
$$

in which

$$
\Gamma(z):=\int_{\gamma} e_{z}(\zeta) n_{q}(\zeta) \ln \left[\left(W(\zeta)-W\left(z_{0}\right)\right)^{-\kappa} G(\zeta)\right] d s, \quad z \notin \gamma,
$$

is a hyperanalytic function in $\mathbb{C} \backslash \gamma$.

Remark about the hypercomplex exponential and logarithmic functions can be found for instance in $[8,9]$.

The following theorem gives characterization for hypercomplex function to admit a canonical factorization. 
THeOREM 3.1. The function $G$ admits a canonical factorization if and only if $\ln [(W(t)-$ $\left.\left.W\left(z_{0}\right)\right)^{-\kappa} G(t)\right] \in \mathscr{S}(\gamma)$.

Proof. Assume $G$ admits a canonical factorization, then we deduce

$$
\ln \left[\left(W(t)-W\left(z_{0}\right)\right)^{-\kappa} G(t) v\right]=\ln H^{+}(t)-\ln \left[\left(W(t)-W\left(z_{0}\right)\right)^{\kappa} H^{-}(t)\right],
$$

for $t \in \gamma$ and $H^{ \pm}$satisfying (3.1).

Now the necessity follows from Lemma 2.4 and the previous equality.

Conversely, suppose $\ln \left[\left(W(t)-W\left(z_{0}\right)\right)^{-\kappa} G(t)\right] \in \mathscr{S}(\gamma)$. Taking into account (3.5) and Theorem 2.5, it is easy to check that $X$ gives a canonical factorization of $G$ on $\gamma$ in the form $G=X^{+} / X^{-}$.

Definition 3.2. $p_{0}+p_{1} W(z)+\cdots+p_{s}(W(z))^{s}, s \geq 0$, is called a hypercomplex polynomial.

Theorem 3.3. Suppose G admits a canonical factorization. For the homogeneous Riemann boundary value problem $(g(t) \equiv 0)$, if $\Phi(\infty)=0$, then it has $\kappa$ linearly independent solutions when $\kappa>0$ and has only the trivial solution when $\kappa \leq 0$. The general solution is given by

$$
\Phi(z)=X(z) P_{\kappa-1}(z)
$$

where $P_{\kappa-1}$ is an arbitrary hypercomplex polynomial whose degree is not greater than $\kappa-1$ $\left(P_{\kappa-1} \equiv 0\right.$ when $\left.\kappa \leq 0\right)$.

Proof. The proof is standard $[1,8,9,10]$ running along similar lines to those in the complex case $[4,5,7,12,13]$ and will not be given here.

Theorem 3.4. Assume $G$ admits a canonical factorization. The Riemann boundary value problem is solvable if and only if $g / X^{+} \in \mathscr{S}(\gamma)$.

Proof. The boundary condition (1.1) may be rewritten as

$$
\frac{\Phi^{+}(t)}{X^{+}(t)}-\frac{\Phi^{-}(t)}{X^{-}(t)}=\frac{g(t)}{X^{+}(t)}, \quad t \in \gamma
$$

The necessary condition is an immediate consequence of (3.7) and Lemma 2.4. Let now $g / X^{+} \in \mathscr{S}(\gamma)$, then obviously the function

$$
X(z)\left(\mathbf{C}_{\gamma}\left\{g / X^{+}\right\}\right)(z)
$$

is a solution of the Riemann boundary value problem.

We consider now $G \in I_{0}(\gamma)$ and $g \in \mathscr{S}(\gamma)$. Note that

$$
\ln \left[\left(W(t)-W\left(z_{0}\right)\right)^{-\kappa} G(t)\right] \in I_{0}(\gamma) \subset \mathscr{Y}(\gamma),
$$

hence, $G$ admits a canonical factorization. 
2834 Riemann boundary value problem for hyperanalytic functions

Taking into account the condition $g \in \mathscr{S}(\gamma)$, it can easily be shown that

$$
\begin{aligned}
\int_{\gamma_{\epsilon}(t)} e_{t}(\zeta) n_{q}(\zeta)\left(\frac{g(\zeta)}{X^{+}(\zeta)}-\frac{g(t)}{X^{+}(t)}\right) d s \\
=\int_{\gamma_{\epsilon}(t)} e_{t}(\zeta) n_{q}(\zeta)\left(\frac{g^{+}(\zeta)}{X^{+}(\zeta)}-\frac{g^{+}(t)}{X^{+}(t)}\right) d s \\
\quad+\frac{1}{G(t)} \int_{\gamma_{\epsilon}(t)} e_{t}(\zeta) n_{q}(\zeta)\left(\frac{g^{-}(\zeta)}{X^{-}(\zeta)}-\frac{g^{-}(t)}{X^{-}(t)}\right) d s \\
\quad+\int_{\gamma_{\epsilon}(t)} e_{t}(\zeta) n_{q}(\zeta)\left(\frac{1}{G(\zeta)}-\frac{1}{G(t)}\right) \frac{g^{-}(\zeta)}{X^{-}(\zeta)} d s=: J_{1}+J_{2}+J_{3}, \quad \in \in(0, d] .
\end{aligned}
$$

In view of the result of Lemma 2.4, $J_{1}, J_{2}$ converge uniformly to zero for $t \in \gamma$, as $\epsilon \rightarrow 0$.

Furthermore, as

$$
\left|J_{3}\right| \leq c \int_{0}^{\epsilon} \frac{\omega_{G}(\xi)}{\xi} d \xi
$$

we have that $g / X^{+} \in \mathscr{Y}(\gamma)$.

Taking into account the above and Theorem 3.4, we thus have proved the following theorem.

Theorem 3.5. Let $G \in I_{0}(\gamma)$ and $g \in \mathscr{S}(\gamma)$. Under the requirement $\Phi(\infty)=0$, the Riemann boundary value problem is solvable when $\kappa \geq 0$ with the general solution

$$
\Phi(z)=X(z)\left(\mathbf{C}_{\gamma}\left\{g / X^{+}\right\}\right)(z)+X(z) P_{\kappa-1}(z) .
$$

Here $P_{\kappa-1}(z)$ is the same as above; when $\kappa<0$, it is (uniquely) solvable with the solution (3.12) $\left(P_{\kappa-1}(z) \equiv 0\right.$ when $\left.\kappa \leq 0\right)$ if and only if the following conditions are satisfied:

$$
\int_{\gamma} \partial_{\zeta} W(\zeta) n_{q}(\zeta) \frac{g(\zeta)}{X^{+}(\zeta)}(W(\zeta))^{k} d s=0, \quad k=0, \ldots,-\kappa-1
$$

\section{Singular integral equations in the class $\mathscr{E}_{\varphi}(\gamma)$}

This section is devoted to the study of the solvability of the singular integral equation of the type

$$
a(t) \Upsilon(t)+b(t)\left(\mathbf{S}_{\gamma} \Upsilon\right)(t)=f(t), \quad t \in \gamma,
$$

for which we assume that $a, b$, and $f$ are given hypercomplex functions. For this equation, $\Upsilon$ is an unknown hypercomplex function belonging to $\mathscr{E}_{\varphi}(\gamma)$.

We assume that $a, b \in \mathscr{H}_{\psi}(\gamma), f \in \mathscr{L}_{\varphi}(\gamma)$, and $a_{0}^{2}(t)-b_{0}^{2}(t) \neq 0$ for $t \in \gamma$. Here $\varphi, \psi$ are given majorants satisfying the following relations:

$$
\begin{gathered}
\epsilon \int_{\epsilon}^{d} \frac{\varphi(\xi)}{\xi^{2}} d \xi \leq c \varphi(\epsilon), \\
\int_{0}^{\epsilon} \frac{\psi(\xi)}{\xi} d \xi \leq c \varphi(\epsilon), \quad \epsilon \in(0, d] .
\end{gathered}
$$


We consider the Riemann boundary value problem associated with the singular integral equation (4.1),

$$
\Phi^{+}(t)-\frac{a(t)-b(t)}{a(t)+b(t)} \Phi^{-}(t)=\frac{f(t)}{a(t)+b(t)}, \quad t \in \gamma, \Phi(\infty)=0
$$

which is similar to (1.1). To simplify notation we write

$$
G(t):=\frac{a(t)-b(t)}{a(t)+b(t)}, \quad g(t):=\frac{f(t)}{a(t)+b(t)}, \quad t \in \gamma
$$

In view of the indicated relation, the index $\kappa$ of the function $\left(a_{0}(t)-b_{0}(t)\right) /\left(a_{0}(t)+b_{0}(t)\right)$, and the corresponding functions $\Gamma(z)$ and $X(z)$, we arrive at the following assertions.

Theorem 4.1. Let $a, b \in \mathscr{H}_{\psi}(\gamma)$ and $f \in \mathscr{L}_{\varphi}(\gamma)$. Then

(i) $G \in \mathscr{H}_{\psi}(\gamma)$,

(ii) $g, X^{ \pm} \in \mathscr{L}_{\varphi}(\gamma)$.

Proof. By straightforward computation, the statement (i) can be obtained. On the other hand, since $\psi(\xi) / \xi$ does not increase,

$$
\psi(\epsilon)=\int_{0}^{\epsilon} \frac{\psi(\epsilon)}{\epsilon} d \xi \leq \int_{0}^{\epsilon} \frac{\psi(\xi)}{\xi} d \xi \leq c \varphi(\epsilon), \quad \epsilon \in(0, d]
$$

we thus have that $g \in \mathscr{H}_{\varphi}(\gamma)$.

Let now

$$
h(t):=(a(t)+b(t))^{-1}, \quad t \in \gamma,
$$

then

$$
\begin{aligned}
& \left|\int_{\gamma_{\epsilon}(t)} e_{t}(\zeta) n_{q}(\zeta)(f(\zeta) h(\zeta)-f(t) h(t)) d s\right| \\
& \leq|h(t)|\left|\int_{\gamma_{\epsilon}(t)} e_{t}(\zeta) n_{q}(\zeta)(f(\zeta)-f(t)) d s\right| \\
& \quad+\left|\int_{\gamma_{\epsilon}(t)} e_{t}(\zeta) n_{q}(\zeta)(h(\zeta)-h(t)) f(\zeta) d s\right| \\
& \leq c\left(\Theta_{f}(\epsilon)+\int_{0}^{\epsilon} \frac{\omega_{h}(\xi)}{\xi} d \xi\right) \\
& \leq c\left(\Theta_{f}(\epsilon)+\int_{0}^{\epsilon} \frac{\psi(\xi)}{\xi} d \xi\right) \leq c \varphi(\epsilon), \quad \epsilon \in(0, d] .
\end{aligned}
$$

Therefore $g \in \mathscr{E}_{\varphi}(\gamma)$. 
2836 Riemann boundary value problem for hyperanalytic functions

We consider now the function $X^{+}$. Suppose $t_{1}, t_{2} \in \gamma$, then

$$
\begin{aligned}
\left|X^{+}\left(t_{1}\right)-X^{+}\left(t_{2}\right)\right| & \leq c\left|\Gamma^{+}\left(t_{1}\right)-\Gamma^{+}\left(t_{2}\right)\right| \\
& \leq c\left(\int_{0}^{\epsilon} \frac{\omega_{\ln G}(\xi)}{\xi} d \xi+\epsilon \int_{\epsilon}^{d} \frac{\omega_{\ln G}(\xi)}{\xi^{2}} d \xi+\omega_{\ln G}(\epsilon)\right) \\
& \leq c\left(\int_{0}^{\epsilon} \frac{\omega_{G}(\xi)}{\xi} d \xi+\epsilon \int_{\epsilon}^{d} \frac{\omega_{G}(\xi)}{\xi^{2}} d \xi+\omega_{G}(\epsilon)\right) \\
& \leq c\left(\int_{0}^{\epsilon} \frac{\psi(\xi)}{\xi} d \xi+\epsilon \int_{\epsilon}^{d} \frac{\psi(\xi)}{\xi^{2}} d \xi+\psi(\epsilon)\right) \leq c \varphi(\epsilon),
\end{aligned}
$$

where $\left|t_{1}-t_{2}\right|=\epsilon$.

Thus, we obtain that $X^{+} \in \mathscr{H}_{\varphi}(\gamma)$. Further

$$
\begin{aligned}
& \left|\int_{\gamma_{\epsilon}(t)} e_{t}(\zeta) n_{q}(\zeta)\left(X^{+}(\zeta)-X^{+}(t)\right) d s\right| \\
& \quad=\left|\int_{\gamma \backslash \gamma_{\epsilon}(t)} e_{t}(\zeta) n_{q}(\zeta)\left(X^{+}(\zeta)-X^{+}(t)\right) d s\right| \\
& \quad \leq c \max _{z \in \bar{\Omega}_{+},|z-t|=\epsilon}\left|X^{+}(z)-X^{+}(t)\right| \\
& \quad \leq c \max _{z \in \Omega_{+},|z-t|=\epsilon}\left|\Gamma^{+}(z)-\Gamma^{+}(t)\right|, \quad \epsilon \in(0, d] .
\end{aligned}
$$

Making use of the Lemma 2.6, we get

$$
\left|\int_{\gamma_{\epsilon}(t)} e_{t}(\zeta) n_{q}(\zeta)\left(X^{+}(\zeta)-X^{+}(t)\right) d s\right| \leq c\left(\omega_{\ln G}(\epsilon)+\Theta_{\ln G}(\epsilon)+\epsilon \int_{\epsilon}^{d} \frac{\omega_{\ln G}(\xi)}{\xi^{2}} d \xi\right)
$$

Taking into account that

$$
\left|\int_{\gamma_{\epsilon}(t)} e_{t}(\zeta) n_{q}(\zeta)(\ln G(\zeta)-\ln G(t)) d s\right| \leq c \int_{0}^{\epsilon} \frac{\psi(\xi)}{\xi} d \xi \leq c \varphi(\epsilon)
$$

we have

$$
\left|\int_{\gamma_{\epsilon}(t)} e_{t}(\zeta) n_{q}(\zeta)\left(X^{+}(\zeta)-X^{+}(t)\right) d s\right| \leq c \varphi(\epsilon)
$$

This implies that $X^{+} \in \mathscr{L}_{\varphi}(\gamma)$, which proves the desired result.

The proof of the assertion concerning $X^{-}$is based on the fact that the function $G$ admits a canonical factorization in the form $G=X^{+} / X^{-}$on $\gamma$. 
Theorem 4.2. Suppose $a, b \in \mathscr{H}_{\psi}(\gamma)$ and $f \in \mathscr{L}_{\varphi}(\gamma)$. Then

(i) $g / X^{+} \in \mathscr{L}_{\varphi}(\gamma)$,

(ii) $X^{ \pm}(t) \int_{\gamma} e_{t}(\zeta) n_{q}(\zeta)\left((g(\zeta)-g(t)) / X^{+}(\zeta)\right) d s \in \mathscr{L}_{\varphi}(\gamma), t \in \gamma$.

Proof. Since $g \in \mathscr{L}_{\varphi}(\gamma) \subset \mathscr{Y}(\gamma)$, then

$$
\begin{aligned}
& \left|\int_{\gamma_{\epsilon}(t)} e_{t}(\zeta) n_{q}(\zeta)\left(\frac{g(\zeta)}{X^{+}(\zeta)}-\frac{g(t)}{X^{+}(t)}\right) d s\right| \\
& \quad \leq\left|\int_{\gamma_{\epsilon}(t)} e_{t}(\zeta) n_{q}(\zeta)\left(\frac{g^{+}(\zeta)}{X^{+}(\zeta)}-\frac{g^{+}(t)}{X^{+}(t)}\right) d s\right| \\
& \quad+\left|\int_{\gamma_{\epsilon}(t)} e_{t}(\zeta) n_{q}(\zeta)\left(\frac{g^{-}(\zeta)}{X^{+}(\zeta)}-\frac{g^{-}(t)}{X^{+}(t)}\right) d s\right|=: J_{1}+J_{2}, \quad \epsilon \in(0, d] .
\end{aligned}
$$

According to Lemma 2.4, we get

$$
\begin{aligned}
J_{1} & \leq c \max _{z \in \Omega_{+},|z-t|=\epsilon}\left|\frac{g^{+}(z)}{X^{+}(z)}-\frac{g^{+}(t)}{X^{+}(t)}\right| \\
& \leq c\left(\max _{z \in \Omega_{+},|z-t|=\epsilon}\left|g^{+}(z)-g^{+}(t)\right|+\max _{z \in \Omega_{+},|z-t|=\epsilon}\left|X^{+}(z)-X^{+}(t)\right|\right) .
\end{aligned}
$$

By Lemma 2.6

$$
\max _{z \in \Omega_{+},|z-t|=\epsilon}\left|g^{+}(z)-g^{+}(t)\right| \leq\left(\omega_{g}(\epsilon)+\Theta_{g}(\epsilon)+\epsilon \int_{\epsilon}^{d} \frac{\omega_{g}(\xi)}{\xi^{2}} d \xi\right) .
$$

Thus, $J_{1} \leq c \varphi(\epsilon)$. Now we estimate $J_{2}$ :

$$
\begin{aligned}
J_{2} \leq & \left|\int_{\gamma_{\epsilon}(t)} e_{t}(\zeta) n_{q}(\zeta)\left(\frac{1}{G(\zeta)}-\frac{1}{G(t)}\right) \frac{g^{-}(\zeta)}{X^{-}(\zeta)} d s\right| \\
& +\left|\frac{1}{G(t)}\right|\left|\int_{\gamma_{\epsilon}(t)} e_{t}(\zeta) n_{q}(\zeta)\left(\frac{g^{-}(\zeta)}{X^{-}(\zeta)}-\frac{g^{-}(t)}{X^{-}(t)}\right) d s\right| \\
& \leq c\left(\int_{0}^{\epsilon} \frac{\omega_{G}(\xi)}{\xi} d \xi+\max _{z \in \Omega_{-},|z-t|=\epsilon}\left|\frac{g^{-}(z)}{X^{-}(z)}-\frac{g^{-}(t)}{X^{-}(t)}\right|\right) \leq c \varphi(\epsilon) .
\end{aligned}
$$

In view of the previous estimations we get immediately that $g / X^{+} \in \mathscr{F}_{\varphi}(\gamma)$.

We now examine the function

$$
\Psi(z):=\left(\mathbf{C}_{\gamma}\left\{g / X^{+}\right\}\right)(z) .
$$


2838 Riemann boundary value problem for hyperanalytic functions

Since $g / X^{+} \in \mathscr{E}_{\varphi}(\gamma)$, then the function

$$
\Psi^{-}(t)=\left(\mathbf{C}_{\gamma}^{-}\left\{g / X^{+}\right\}\right)(t)=\int_{\gamma} e_{t}(\zeta) n_{q}(\zeta)\left(\frac{g(\zeta)}{X^{+}(\zeta)}-\frac{g(t)}{X^{+}(t)}\right) d s \in \mathscr{E}_{\varphi}(\gamma) .
$$

Now by applying Lemma 2.4, we also obtain that

$$
\begin{aligned}
\Psi^{-}(t)= & \left(\mathbf{C}_{\gamma}^{-}\left\{g / X^{+}\right\}\right)(t) \\
= & \int_{\gamma} e_{t}(\zeta) n_{q}(\zeta)\left(\frac{g(\zeta)-g(t)}{X^{+}(\zeta)}\right) d s \\
& +g(t) \int_{\gamma} e_{t}(\zeta) n_{q}(\zeta)\left(\frac{1}{X^{+}(\zeta)}-\frac{1}{X^{+}(t)}\right) d s \\
= & \int_{\gamma} e_{t}(\zeta) n_{q}(\zeta)\left(\frac{g(\zeta)-g(t)}{X^{+}(\zeta)}\right) d s \in \mathscr{E}_{\varphi}(\gamma),
\end{aligned}
$$

consequently $X^{+} \Psi^{-} \in \mathscr{H}_{\varphi}(\gamma)$.

On the other hand,

$$
\begin{aligned}
& \left|\int_{\gamma_{\epsilon}(t)} e_{t}(\zeta) n_{q}(\zeta)\left(X^{+}(\zeta) \Psi^{-}(\zeta)-X^{+}(t) \Psi^{-}(t)\right) d s\right| \\
& =\mid \int_{\gamma_{\epsilon}(t)} e_{t}(\zeta) n_{q}(\zeta)(G(\zeta)-G(t)) X^{-}(\zeta) \Psi^{-}(\zeta) d s \\
& \quad+G(t) \int_{\gamma_{\epsilon}(t)} e_{t}(\zeta) n_{q}(\zeta)\left(X^{-}(\zeta) \Psi^{-}(\zeta)-X^{-}(t) \Psi^{-}(t)\right) d s \mid \\
& \quad \leq c\left(\int_{0}^{\epsilon} \frac{\omega_{G}(\xi)}{\xi} d \xi+\left|\int_{\gamma_{\epsilon}(t)} e_{t}(\zeta) n_{q}(\zeta)\left(X^{-}(\zeta) \Psi^{-}(\zeta)-X^{-}(t) \Psi^{-}(t)\right) d s\right|\right) \\
& \leq c \varphi(\epsilon), \quad \epsilon \in(0, d],
\end{aligned}
$$

hence, $X^{+} \Psi^{-} \in \mathscr{L}_{\varphi}(\gamma)$.

Finally, since $X^{-} \Psi^{-}=\left(X^{+} \Psi^{-}\right) / G$, then $X^{-} \Psi^{-} \in \mathscr{L}_{\varphi}(\gamma)$, which completes the proof.

We can now state our final result.

Theorem 4.3. Let $a, b \in \mathscr{H}_{\psi}(\gamma)$. If $\kappa \geq 0$, the singular integral equation (4.1) is solvable in the class $\mathscr{E}_{\varphi}(\gamma)$ for all $f \in \mathscr{L}_{\varphi}(\gamma)$; if $\kappa<0$, then this equation is solvable in the class $\mathscr{L}_{\varphi}(\gamma)$ if and only if the following conditions are satisfied:

$$
\int_{\gamma} \partial_{\zeta} W(\zeta) n_{q}(\zeta)\left(\frac{f(\zeta)}{(a(\zeta)+b(\zeta)) X^{+}(\zeta)}\right)(W(\zeta))^{k} d s=0, \quad k=0, \ldots,-\kappa-1
$$


The general solution of (4.1) in the class $\mathscr{E}_{\varphi}(\gamma)$ has the form

$$
\begin{aligned}
\Upsilon(t)= & \left(X^{+}(t)-X^{-}(t)\right) \int_{\gamma} e_{t}(\zeta) n_{q}(\zeta)\left(\frac{g(\zeta)-g(t)}{X^{+}(\zeta)}\right) d s \\
& +g(t)+\left(X^{+}(t)-X^{-}(t)\right) P_{\kappa-1}(t), \quad t \in \gamma,
\end{aligned}
$$

where $P_{\kappa-1}$ is an arbitrary hypercomplex polynomial whose degree is not greater than $\kappa-1$ for $\kappa>0$ and $P_{\kappa-1} \equiv 0$ for $\kappa \leq 0$.

Proof. The proof follows directly by using Theorems 3.5, 4.1, and 4.2.

Acknowledgments. The research carried out in this paper was initiated while the third author was visiting the Department of Mathematics, University of Oriente, Santiago de Cuba. It was completed during his stay as a Ph.D. student at the Department of Mathematical Analysis, Clifford Research Group, Ghent University, supported by the Special Research Fund of Ghent University under the Doctoral Grant 011/DS 503. He would like to express his sincere gratitude.

\section{References}

[1] H. Begehr, Complex Analytic Methods for Partial Differential Equations. An Introductory Text, World Scientific, New Jersey, 1994.

[2] H. Begehr and R. P. Gilbert, On Riemann boundary value problems for certain linear elliptic systems in the plane, J. Differential Equations 32 (1979), no. 1, 1-14.

[3] R. A. Blaya, D. P. Peña, and J. B. Reyes, Conjugate hyperharmonic functions and Cauchy type integrals in Douglis analysis, Complex Variables Theory Appl. 48 (2003), no. 12, 1023-1039.

[4] J. Bustamante González and B. González Diéguez, The Riemann boundary value problem and singular integral equations on closed nonsmooth curves, Cienc. Mat. (Havana) 11 (1990), no. 1, 13-16 (Spanish).

[5] R. Estrada and R. P. Kanwal, The Carleman type singular integral equations, SIAM Rev. 29 (1987), no. 2, 263-290.

[6] Singular Integral Equations, Birkhäuser Boston, Massachusetts, 2000.

[7] F. D. Gakhov, Boundary Value Problems, 3rd ed., Izdat. "Nauka", Moscow, 1977, revised and augmented (Russian).

[8] R. P. Gilbert and J. L. Buchanan, First Order Elliptic Systems. A Function Theoretic Approach, Mathematics in Science and Engineering, vol. 163, Academic Press, Florida, 1983.

[9] R. P. Gilbert and G. N. Hile, Generalized hypercomplex function theory, Trans. Amer. Math. Soc. 195 (1974), 1-29.

[10] R. P. Gilbert and Y. S. Zeng, Hyperanalytic Riemann boundary value problems on rectifiable closed curves, Complex Variables Theory Appl. 20 (1992), no. 1-4, 277-288.

[11] B. González Diéguez and J. Bustamante González, Invariance of a class of functions under the action of a singular integral operator, Cienc. Mat. (Havana) 6 (1985), no. 3, 41-45 (Spanish).

[12] J. K. Lu, Boundary Value Problems for Analytic Functions, Series in Pure Mathematics, vol. 16, World Scientific, New Jersey, 1993.

[13] N. I. Muskhelishvili, Singular Integral Equations: Boundary Value Problems in the Theory of Function and Their Applications of Them to Mathematical Physics, Izdat. "Nauka", Moscow, 1968 , 3rd corrected and augmented ed., with an appendix by B. Bojarski. 


\section{Riemann boundary value problem for hyperanalytic functions}

[14] S. A. Plaksa, On the Noethericity of singular integral equations with a Cauchy kernel on a rectifiable curve, Ukraïn. Mat. Zh. 45 (1993), no. 10, 1379-1389 (Russian), translated in Ukrainian Math. J. 45 (1993), no. 10, 1548-1559.

[15] I. N. Vekua, Generalized Analytic Functions, Pergamon Press, London; Addison-Wesley, Massachusetts, 1962.

[16] G. C. Wen and H. Begehr, Boundary Value Problems for Elliptic Equations and Systems, Pitman Monographs and Surveys in Pure and Applied Mathematics, vol. 46, Longman Scientific \& Technical, Harlow; copublished in the United States with John Wiley \& Sons, New York, 1990.

[17] W. L. Wendland, Elliptic Systems in the Plane, Monographs and Studies in Mathematics, vol. 3, Pitman (Advanced Publishing Program), Massachusetts, 1979.

[18] Y. S. Zeng, Riemann boundary value problems for hyperanalytic functions on rectifiable Jordan closed curves, Hunan Ann. Math. 11 (1991), no. 1-2, 31-38 (Chinese).

Ricardo Abreu Blaya: Faculty of Mathematics and Informatics, University of Holguín, Holguín 80100, Cuba

E-mail address: rabreu@facinf.uho.edu.cu

Juan Bory Reyes: Department of Mathematics, University of Oriente, Santiago de Cuba 90500, Cuba

E-mail address: jbory@rect.uo.edu.cu

Dixan Peña Peña: Department of Mathematical Analysis, Ghent University, Galglaan 2, 9000 Ghent, Belgium

E-mail address:dixan@cage.ugent.be 


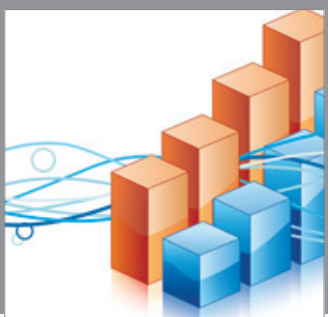

Advances in

Operations Research

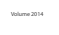

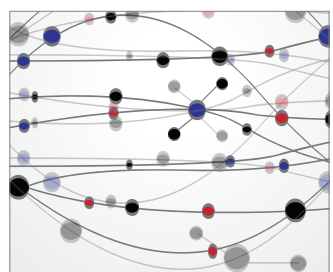

\section{The Scientific} World Journal
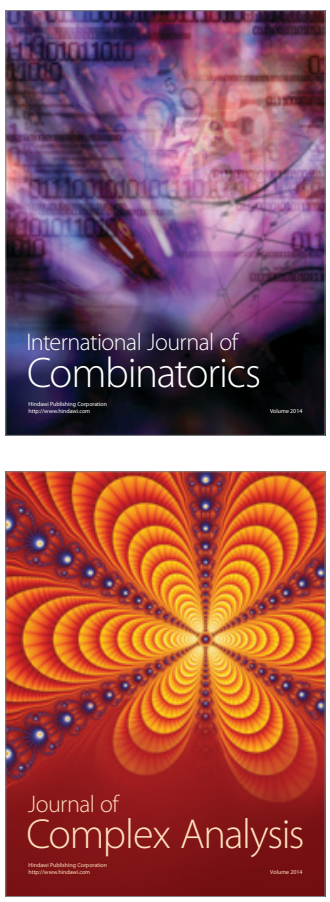

International Journal of

Mathematics and

Mathematical

Sciences
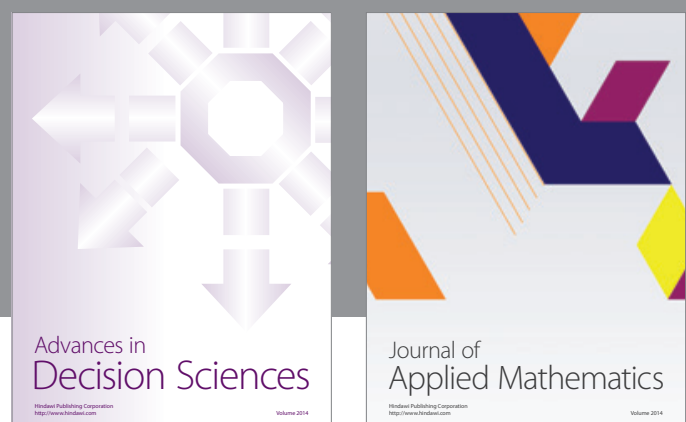

Journal of

Applied Mathematics
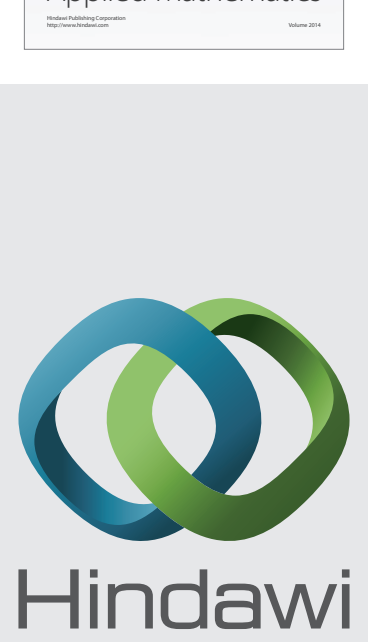

Submit your manuscripts at http://www.hindawi.com
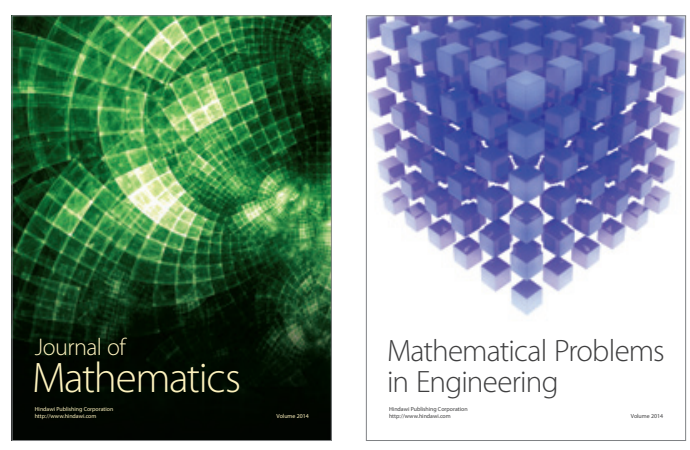

Mathematical Problems in Engineering
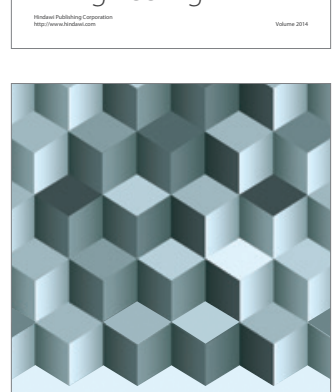

Journal of

Function Spaces
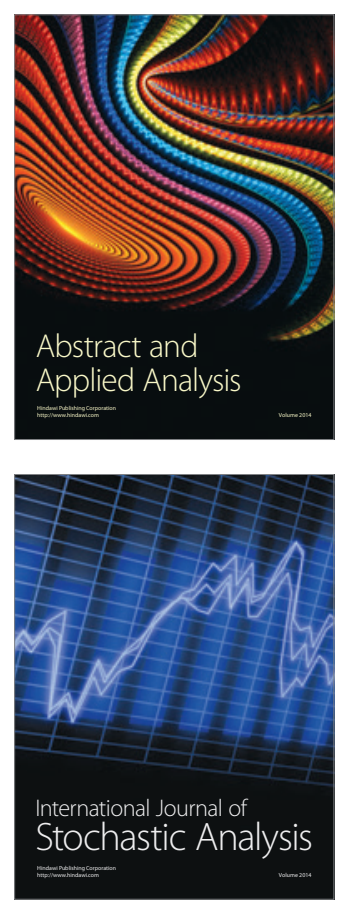

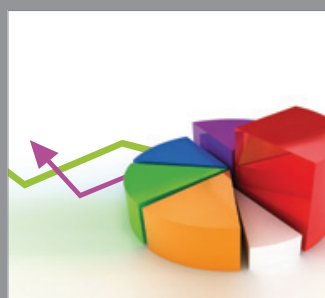

ournal of

Probability and Statistics

Promensencen
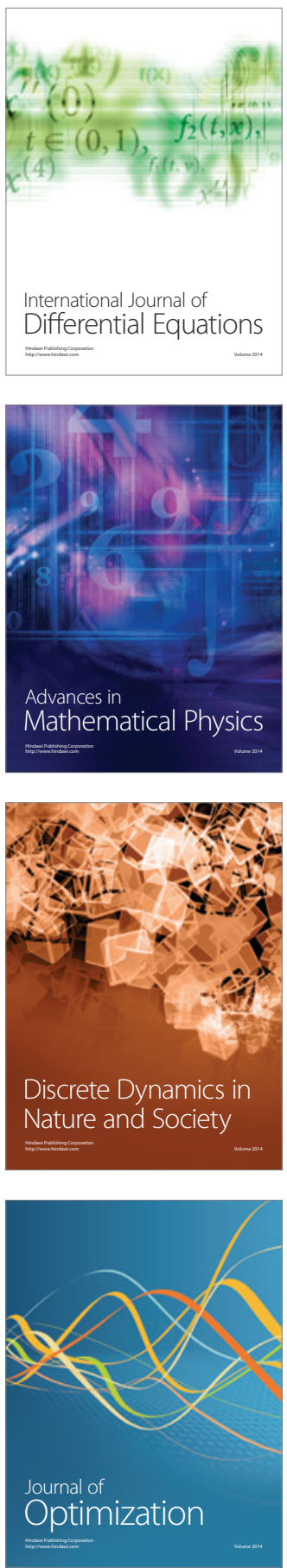